\title{
NO POLYNOMIAL BOUND FOR THE CHIP FIRING GAME ON DIRECTED GRAPHS
}

\author{
KIMMO ERIKSSON \\ (Communicated by Thomas H. Brylawski)
}

\begin{abstract}
Tardos has proved a polynomial bound on the length of a convergent chip firing game on an undirected graph. This paper demonstrates a game with exponential growth on a directed graph.
\end{abstract}

\section{INTRODUCTION}

The chips game for general graphs was formulated by Björner, Lovász, and Shor [1]. For undirected graphs, G. Tardos [2] gave an upper limit, $O\left(n^{4}\right)$, on the number of moves in a game for which the number of nodes is bounded by $n$. In this note we prove that for directed graphs no polynomial bound on the number of moves exists. In fact, we give an example of a sequence of games with exponential growth.

Definitions. The chips game is played in the following way. Place a pile of chips on each node of a connected finite graph. A move now consists of selecting a node with at least as many chips in its pile as it has outgoing edges, and firing that node by moving one chip to each of its neighbors (respecting the direction of edges). Consequently, a position where each node has too few chips to be fired is a final position. This game is known to be strongly convergent [1], that is, either every legal game from the initial position is infinite or every legal game terminates with the same final position in the same number of moves, whatever choices have been made during play.

The exponential game. We define a game for every even positive number $n$. Take a bidirected circuit of $n-1$ nodes and add a center node with edges to all other nodes, all but one bidirected, the last one directed from the center node. The initial position is $3 n-5$ chips on the center node and no chips on the circuit nodes. This game is convergent, as will be seen later.

Only one distribution of $3 n-5$ chips will do as a final position: $n-2$ on the center node, 1 on the node with the directed edge (call this node the top node), and 2 on all the others.

Received by the editors July 29, 1989.

1980 Mathematics Subject Classification (1985 Revision). Primary 05C35.

Key words and phrases. Graph, game, chip firing. 


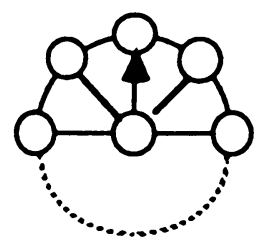

FIGURE 1. The graph.

We want to compute the length of this game. Observe that if there is at least one chip on every circuit node then the following sequence of moves is legal: fire the center node, the top node, and clockwise the other circuit nodes. In this operation every node is fired once, transporting one chip from the center node to the top node. Firing all nodes once again followed by an additional firing of the top node results in a net flow of two chips from the center node, one to each of the neighbors of the top node. This operation, consisting of $2 n+1$ moves, can be done over and over again with a few intermediate moves, thereby constructing the total solution of the game.

Starting from the initial position, let us look at the circuit nodes near the top node.

Fire the center node

$2 n+1$ moves

$\cdots 1111111 \cdots \quad\left(P_{0}\right)$

$2 n+1$ moves

$\cdots 1121211 \cdots \quad\left(P_{1}\right)$

Fire the top node's neighbors and the top node

$\cdots 1131311 \ldots$

$2 n+1$ moves

$\cdots 1211121 \cdots$

$\cdots 1221221 \cdots \quad\left(P_{2}\right)$

In this way we can create the whole game by induction. Let $P_{i}$ denote a $\cdots 12 \cdots 212 \cdots 21 \cdots$ position, with $i$ twos on each side of the top node. Assume that for $i=1,2, \ldots, k-1$ we know sequences of $a_{i}$ moves leading from $P_{i-1}$ to $P_{i}$. Play from $P_{k-1}$ to $P_{k}$ :

$$
a_{k-1} \text { moves } \quad \cdots 132 \cdots 212 \cdots 231 \cdots
$$

Fire all nodes from left 3 to right $3 \cdots 211 \cdots 111 \cdots 112 \cdots$

( $2 k-1$ moves)

Rebuild the inner twos,

$$
\sum a_{i} \text { moves, } i=1,2, \ldots, k-1 \quad \cdots 222 \cdots 212 \cdots 222 \cdots \quad\left(P_{k}\right)
$$

Letting $a_{k}$ be the number of moves from $P_{k-1}$ to $P_{k}$ we get a recurrence:

$$
a_{k}=a_{k-1}+(2 k-1)+\sum_{i=1}^{k-1} a_{i}, \quad \text { for } k \geq 2, a_{1}=2 n+1 .
$$

Let $s_{k}$ be the total number of moves from the initial position to $P_{k}$. Then 


$$
\begin{aligned}
& s_{k}=1+\sum_{i=1}^{k} a_{i} \text {, so } \\
& \qquad s_{k}-3 s_{k-1}+s_{k-2}=2 k-2 \text { for } k \geq 2, s_{0}=1, s_{1}=2 n+2 .
\end{aligned}
$$

This recurrence has the solution

$$
s_{k}=A\left(\frac{1+\sqrt{5}}{2}\right)^{2 k}+B\left(\frac{1+\sqrt{5}}{2}\right)^{-2 k}-2 k,
$$

and $s_{0}$ and $s_{1}$ give

$$
A=\frac{2 n}{\sqrt{5}}+\frac{1+\sqrt{5}}{2}, \quad B=-\frac{2 n}{\sqrt{5}}-\frac{2}{1+\sqrt{5}} .
$$

The final position is achieved when all nodes except the top node and the center node has two chips, i.e., after $s_{m}$ moves, with $m=(n-2) / 2$. With $\tau=$ $(1+\sqrt{5}) / 2$ we have, asymptotically,

$$
s_{m} \sim(2 n / \sqrt{5}+\tau) \tau^{(n-2)},
$$

clearly showing that the growth is exponential.

\section{REFERENCES}

1. A. Björner, L. Lovász, and P. Shor, Chip-firing games on graphs, preprint, 1987.

2. G. Tardos, Polynomial bound for a chip firing game on graphs, SIAM J. Discrete Math. 1 (1988), 397-398.

Department of Mathematics, Royal Institute of Technology, S-100 44 Stockholm, SWEDEN 